\title{
Game Theoretic Strategies for Spacecraft Rendezvous and Motion Synchronization
}

\author{
Vincenzo Tartaglia ${ }^{1}$ and Mario Innocenti ${ }^{2}$ \\ University of Pisa, 56122 Pisa, Italy
}

\begin{abstract}
The rendezvous problem between two active spacecraft is formulated as a two player nonzero-sum differential game. The local-vertical local-horizontal (LVLH) rotating reference frame is used to describe the dynamics of the game. Linear quadratic cooperative and noncooperative differential games are applied to obtain a feedback control law. A comparison between Pareto and Nash equilibria was then performed. The state-dependent Riccati equation (SDRE) method is applied to extend the Linear Quadratic differential game theory to obtain a feedback controller in the case of nonlinear relative motion dynamics. Finally, a multiplayer sequential game strategy is synthesized to extend the control law to the relative motion synchronization of multiple vehicles.
\end{abstract}

\section{Introduction}

$\mathrm{R}^{\mathrm{s}}$ ENDEZVOUS problems between spacecraft have attracted high research interest due to their important applications in future space missions. Rendezvous problems can be categorized into two groups depending on how the rendezvous maneuver is performed by the participating spacecraft for a given mission: active-passive and cooperative maneuvers. In an active-passive rendezvous problem involving two spacecraft, the first spacecraft, known as the target, does not apply any control force while following its trajectory. The second spacecraft, which serves as an active spacecraft, is controlled in order to meet the target. The control objective for the active spacecraft is matching the position and the velocity of the target spacecraft. On the other hand, in a cooperative rendezvous maneuver, both spacecraft are controlled and guided to match the same position and velocity. Therefore, the solutions to both rendezvous problems consist of a sequence of control maneuvers or guidance laws, designed to bring the spacecraft to the same states, i.e., position and velocity. Based on the overall mission requirements, the control objective of the rendezvous can include additional constraints such as the total amount of propellant for each spacecraft or the mission time.

The cooperative maneuver is formulated as two-player nonzero-sum differential game. In a nonzero-sum differential game the sum of the objective functions is not equal to zero. Two possible strategies can be used: noncooperative and cooperative. The typical solution of the noncooperative game is the well-known Nash equilibrium. Nash equilibrium can be interpreted as a state where no player has interest to unilaterally deviate from its strategy.

A typical solution of the cooperative game is called Pareto efficient solution. The so-called Pareto efficient solutions are based on the assumption that the cost any one specific player incurs is not uniquely determined, rather the solution is determined when the cost incurred by all players simultaneously cannot be improved.

For nonlinear dynamics the analytical solution of the two players game is complicated by the resolution of Hamilton-Jacobi-Isaacs (HJI) partial differential equations. For a two-player zero-sum Linear Quadratic (LQ) differential game the solution is reduced to the solution of matrix Riccati differential equations. The state-dependent Riccati equation (SDRE) technique is a method to solve the infinite horizon nonlinear differential game.

In this work, a SDRE method is used to solve a nonzero-sum differential game for the spacecraft proximity operations and rendezvous. The dynamics of the spacecraft is expressed in the LVLH frame centered at an actual or a fictions spacecraft in an unperturbed orbit around the Earth. Both spacecraft use a continuous thrust engine and the control is computed by varying the thrust magnitude and direction. The mass of the spacecraft varies with the propellant consumption. A game time interval is fixed a priori and a perfect information structure is assumed.

\footnotetext{
${ }^{1}$ Graduate Student, Department of Information Engineering, Largo Lucio Lazzarino 2.

${ }^{2}$ Full Professor, Department of Information Engineering, Largo Lucio Lazzarino 2, Associate Fellow AIAA. 1 


\section{Relative Motion Equations}

The motion of a deputy spacecraft relative to a chief spacecraft can be described with respect to the local-vertical local-horizontal (LVLH) rotating reference frame [1]. The origin of the LVLH frame is attached to the chief's center of mass. Its orientation is given by the unit vectors $\hat{\boldsymbol{i}}=\boldsymbol{r} / r, \hat{\boldsymbol{j}}=\hat{\boldsymbol{k}} \times \hat{\boldsymbol{i}}$, where $\boldsymbol{h}=\boldsymbol{r} \times \boldsymbol{v}$ is the chief's massless angular momentum vector while $r$ and $\boldsymbol{v}$ are the chief's position and the absolute velocity vectors in the inertial reference frame (see Fig. 1). By denoting the position vector of the deputy, relative to the chief, with $\boldsymbol{\rho}=\left[\begin{array}{lll}x & y & z\end{array}\right]^{\mathrm{T}}$ the nonlinear equations of the relative motion (NERM), under the assumption of Keplerian twobody problem, can be written as:

$$
\left\{\begin{array}{l}
\ddot{x}=2 \dot{f}\left(\dot{y}-\frac{\dot{r}}{r} y\right)+\dot{f}^{2} x+\frac{\mu}{r^{2}}-\frac{\mu}{r_{d}^{3}}(r+x)+u_{x} \\
\ddot{y}=-2 \dot{f}\left(\dot{x}-\frac{\dot{r}}{r} x\right)+\dot{f}^{2} y-\frac{\mu}{r_{d}^{3}} y+u_{y} \\
\ddot{z}=-\frac{\mu}{r_{d}^{3}} z+u_{z}
\end{array}\right.
$$

where $\mu$ is the Earth's gravitational parameter, $r_{D}=\sqrt{(r+x)^{2}+y^{2}+z^{2}}$ is deputy distance from Earth, $f$ is the chief's true anomaly, and its time derivative is given by $\dot{f}=h / r^{2}$. The system can be written in state space form, nonlinear in the state, and affine in the control as:

$$
\dot{\boldsymbol{x}}=f(\boldsymbol{x})+B \boldsymbol{u}(t), \quad B=\left[\begin{array}{c}
0_{3 \times 3} \\
I_{3 \times 3}
\end{array}\right]
$$

where: $\boldsymbol{x}=\left[\begin{array}{ll}\boldsymbol{\rho} & \dot{\boldsymbol{\rho}}\end{array}\right]^{T}=\left[\begin{array}{llllll}x & y & z & \dot{x} & \dot{y} & \dot{z}\end{array}\right]^{T}, \boldsymbol{u}=\left[\begin{array}{lll}u_{x} & u_{y} & u_{z}\end{array}\right]^{T}$.

\section{A. Linearized Equations of Relative Motion}

The NERM can be simplified through linearization around the origin of the chief orbit, and assuming that the relative orbit coordinates are smaller than the chief orbit radius, i.e. $\rho<<r$. The relative motion, under this assumption, can then be approximated by using the linear equations of the relative motion (LERM):

$$
\left\{\begin{array}{l}
\ddot{x}=\dot{f}^{2}\left(1+2 \frac{r}{p}\right) x+2 \dot{f}\left(\dot{y}-\frac{\dot{r}}{r} y\right)+u_{x} \\
\ddot{y}=-2 \dot{f}\left(\dot{x}-\frac{\dot{r}}{r} x\right)+\dot{f}^{2}\left(1-\frac{r}{p}\right) y+u_{y} \\
\ddot{z}=-\frac{r}{p} \dot{f}^{2} z+u_{z}
\end{array}\right.
$$

in state space form Eq. (3) becomes:

$$
\dot{\boldsymbol{x}}_{L}=A_{L}(t) \boldsymbol{x}_{L}+B \boldsymbol{u}=\left[\begin{array}{cc}
0 & I \\
A_{\dot{\rho} \rho}^{L} & A_{\dot{\rho} \dot{\rho}}^{L}
\end{array}\right] \boldsymbol{x}_{L}+B u(t)
$$

where: 


$$
A_{\dot{\rho} \rho}^{L}=\left[\begin{array}{ccc}
\dot{f}^{2}\left(1+2 \frac{r}{p}\right) & -2 \dot{f} \frac{\dot{r}}{r} & 0 \\
2 \dot{f} \frac{\dot{r}}{r} & \dot{f}^{2}\left(1-\frac{r}{p}\right) & 0 \\
0 & 0 & -\dot{f}^{2} \frac{r}{p}
\end{array}\right], \quad A_{\dot{\rho} \dot{\rho}}^{L}=\left[\begin{array}{ccc}
0 & 2 \dot{f} & 0 \\
-2 \dot{f} & 0 & 0 \\
0 & 0 & 0
\end{array}\right]
$$

\section{B. Hill-Clohessly-Wiltshire Equations}

A further simplification can be obtained assuming that the chief's orbit be circular in Eq. (3). The chief's orbit radius $r$ and the true anomaly rate $\dot{f}$ become constant, and the relative equations of motion can be reduced to a simple form known as Hill-Clohessy-Wiltshire (HCW) equations,

$$
\left\{\begin{array}{l}
\ddot{x}=3 n^{2} x+2 n \dot{y}+u_{x} \\
\ddot{y}=-2 n \dot{x}+u_{y} \\
\ddot{z}=-n^{2} z+u_{z}
\end{array}\right.
$$

where $n$ represents the chief's mean motion. The HCW equations in state-space form are expressed below:

$$
\dot{\boldsymbol{x}}_{H}=\left[\begin{array}{cc}
0 & I \\
A_{\dot{\rho} \rho}^{H} & A_{\dot{\rho} \dot{\rho}}^{H}
\end{array}\right] \boldsymbol{x}_{H}+B \boldsymbol{u}
$$

where

$$
A_{\dot{\rho} \rho}^{H}=\left[\begin{array}{ccc}
3 n^{2} & 0 & 0 \\
0 & 0 & 0 \\
0 & 0 & -n^{2}
\end{array}\right], A_{\dot{\rho} \dot{\rho}}^{H}=\left[\begin{array}{ccc}
0 & 2 n & 0 \\
-2 n & 0 & 0 \\
0 & 0 & 0
\end{array}\right]
$$

For a chief spacecraft with nonzero eccentricity, HCW equations only provide an approximate solutions for $\rho$ and $\dot{\rho}$.

\section{Virtual Chief Equations}

In the virtual chief (VC) method [2], a fictional satellite with zero eccentricity is used as the chief satellite for the HCW equations, with both the actual chief and deputy satellites treated as deputies. This virtual chief satellite is a circularized version of the actual chief satellite, and it is defined by setting its eccentricity to zero and all orbital element values equals to those of the chief. The VC method replaces the HCW assumption that the chief is in a circular orbit with the assumption that the chief is in a close proximity of a circular orbit. The VC equations in statespace form are given below:

$$
\dot{\boldsymbol{x}}_{V C}=\left[\begin{array}{cc}
0 & I \\
A_{\rho \rho}^{V C} & A_{\dot{\rho} \dot{\rho}}^{V C}
\end{array}\right] \boldsymbol{x}_{V C}+B \boldsymbol{u}
$$

where, introducing the chief's mean anomaly $M$, 


$$
A_{\dot{\rho} \rho}^{V C}=\left[\begin{array}{ccc}
\dot{f}^{2}+n^{2}\left(3 \cos ^{2}(f-M)-1\right) & \ddot{f}-\frac{3}{2} n^{2} \sin (2(f-M)) & 0 \\
-\ddot{f}-\frac{3}{2} n^{2} \sin (2(f-M)) & \dot{f}^{2}+n^{2}\left(3 \sin ^{2}(f-M)-1\right) & 0 \\
0 & 0 & -n^{2}
\end{array}\right], A_{\dot{\rho} \dot{\rho}}^{V C}=A_{\dot{\rho} \dot{\rho}}^{L}
$$

The out-of-plane components for the VC and HCW equations are identical. For the in-plane components, the VC equations can be considered as an approximation of the LERM with low eccentricity value. This fact will be used in the last section simulation.

\section{Nonzero-Sum Linear Quadratic Differential Games}

Game theory was originated from economics [3], and widely applied in many technological areas such as control system engineering, military and aerospace systems, power systems, communication networks and biomedical science, among others. Game theory deals with strategic interactions among multiple decision makers called players or, in some contexts, agents. The player's objective is captured in a cost function, which the players either minimize or maximize. The decision is called the player's strategy. In this paper, the so-called linear quadratic (LQ) differential games are considered, and the solution of cooperative and noncooperative nonzero-sum differential structures are used in the feedback form as applied to spacecraft relative motion control. Optimal control theory plays an important role in order to solve differential games, the background used here can be found in [4], [5], [6].

\section{A. Cooperative LQ Differential Game}

In a two-player cooperative differential game, Player 1 and Player 2 cooperate in order to achieve their objectives. In case of the infinite-horizon, the objective of each player is the minimization of the their own quadratic cost function. Using standard notation:

$$
\begin{aligned}
& J_{1}\left(\boldsymbol{x}_{0}, \boldsymbol{u}_{1}\right)=\int_{0}^{\infty}\left[\boldsymbol{x}^{\mathrm{T}}(t) Q_{1} \boldsymbol{x}(t)+\boldsymbol{u}_{1}^{\mathrm{T}}(t) R_{1} \boldsymbol{u}_{1}(t)\right] d t \\
& J_{2}\left(\boldsymbol{x}_{0}, \boldsymbol{u}_{2}\right)=\int_{0}^{\infty}\left[\boldsymbol{x}^{\mathrm{T}}(t) Q_{2} \boldsymbol{x}(t)+\boldsymbol{u}_{2}^{\mathrm{T}}(t) R_{2} \boldsymbol{u}_{2}(t)\right] d t
\end{aligned}
$$

subject to the dynamic constraints:

$$
\dot{\boldsymbol{x}}(t)=A \boldsymbol{x}(t)+B_{1} \boldsymbol{u}_{1}(t)+B_{2} \boldsymbol{u}_{2}(t), \boldsymbol{x}(0)=\boldsymbol{x}_{0}
$$

where the weighting matrices $Q_{1}, Q_{2} \in \mathbb{R}^{n \times n}$ are assumed to be symmetric positive semidefinite, and $R_{1} \in \mathbb{R}^{m_{1} \times m_{1}}, R_{2} \in \mathbb{R}^{m_{2} \times m_{2}}$ are symmetric, positive definite. The objectives in Eq. (11) could be possibly conflicting. The players can communicate and can also enter into binding agreements. It is assumed here that each player has all the information about the state dynamics and cost functions of its opponent. The cooperative optimization problem (11)-(12) can be written as a multi-objective optimization problem:

$$
\min _{u} J\left(\boldsymbol{x}_{0}, \boldsymbol{u}\right)=\left[J_{1}\left(\boldsymbol{x}_{0}, \boldsymbol{u}_{1}\right), J_{2}\left(\boldsymbol{x}_{0}, \boldsymbol{u}_{2}\right)\right]
$$

subject to Eq. (12). In cooperative games, Pareto efficient solutions are based on the assumption that the cost one specific player incurs, is not uniquely determined and, depending on how the players choose to divide their control effort, a player can incur in different minima. A set of control strategies $\hat{\boldsymbol{u}}=\left(\hat{\boldsymbol{u}}_{1}, \hat{\boldsymbol{u}}_{2}\right) \in U$ is called Pareto efficient if the set of inequalities holds: 


$$
\begin{aligned}
& J_{1}\left(\boldsymbol{x}_{0}, \boldsymbol{u}_{1}\right) \leq J_{1}\left(\boldsymbol{x}_{0}, \hat{\boldsymbol{u}}_{1}\right) \\
& J_{2}\left(\boldsymbol{x}_{0}, \boldsymbol{u}_{2}\right) \leq J_{2}\left(\boldsymbol{x}_{0}, \hat{\boldsymbol{u}}_{2}\right)
\end{aligned}
$$

Existence of a solution yields $J\left(\boldsymbol{x}_{0}, \hat{\boldsymbol{u}}\right)=\left[J_{1}\left(\boldsymbol{x}_{0}, \hat{\boldsymbol{u}}_{1}\right), J_{2}\left(\boldsymbol{x}_{0}, \hat{\boldsymbol{u}}_{2}\right)\right] \in \mathbb{R}^{2}$ and is called a Pareto solution [7], [8]. In case of the two players, the Pareto solution has a simple characterization depending on the set of control parameters:

$$
A:=\left\{\alpha=\left(\alpha_{1}, \alpha_{2}\right) \mid \alpha_{1}, \alpha_{2} \geq 0, \quad \alpha_{1}+\alpha_{2}=1\right\}
$$

The set of all cooperative Pareto solutions for the optimization problem in Eq. (13) is then given by: $\left(J_{1}\left(\boldsymbol{u}^{*}(\alpha)\right), J_{2}\left(\boldsymbol{u}^{*}(\alpha)\right)\right)$ where $\alpha \in A$. The corresponding Pareto-efficient strategy is obtained as:

$\boldsymbol{u}^{*}(\alpha)=\underset{u \in U}{\operatorname{argmin}}\left[\alpha J_{1}+(1-\alpha) J_{2}\right]$ subject to $\dot{\boldsymbol{x}}(t)=A \boldsymbol{x}(t)+B_{1} \boldsymbol{u}_{1}(t)+B_{2} \boldsymbol{u}_{2}(t)$. In order to find all cooperative solutions for the cooperative LQ differential game, an optimal linear regulator problem must be solved, which depends on a parameter $\alpha$. The so-called Bargaining theory is the theory thanks to which it is possible to choose the best Pareto solution [8]. Bargaining Theory deals with the cases in which the players can realize, through the cooperation, other outcomes better than the ones achievable i in case of no cooperation.

\section{B. Noncooperative LQ Differential Game}

In noncooperative nonzero-sum LQ differential games, the objective of each player is to minimize their own quadratic cost function:

subject to:

$$
\begin{aligned}
& J_{1}\left(\boldsymbol{x}_{0}, \boldsymbol{u}_{1}, \boldsymbol{u}_{2}\right)=\int_{0}^{\infty}\left[\boldsymbol{x}^{\mathrm{T}}(t) Q_{1} \boldsymbol{x}(t)+\boldsymbol{u}_{1}^{\mathrm{T}}(t) R_{11} \boldsymbol{u}_{1}(t)+\boldsymbol{u}_{2}^{\mathrm{T}}(t) R_{12} \boldsymbol{u}_{2}(t)\right] d t \\
& J_{2}\left(\boldsymbol{x}_{0}, \boldsymbol{u}_{1}, \boldsymbol{u}_{2}\right)=\int_{0}^{\infty}\left[\boldsymbol{x}^{\mathrm{T}}(t) Q_{2} \boldsymbol{x}(t)+\boldsymbol{u}_{1}^{\mathrm{T}}(t) R_{21} \boldsymbol{u}_{1}(t)+\boldsymbol{u}_{2}^{\mathrm{T}}(t) R_{22} \boldsymbol{u}_{2}(t)\right] d t
\end{aligned}
$$

$$
\dot{\boldsymbol{x}}(t)=A \boldsymbol{x}(t)+B_{1} \boldsymbol{u}_{1}(t)+B_{2} \boldsymbol{u}_{2}(t), \boldsymbol{x}(0)=\boldsymbol{x}_{0}
$$

where the weighting matrices $Q_{1}, Q_{2} \in \mathbb{R}^{n \times n}, R_{21} \in \mathbb{R}^{m_{1} \times m_{1}}, R_{12} \in \mathbb{R}^{m_{2} \times m_{2}}$ are symmetric positive semidefinite, and $R_{11} \in \mathbb{R}^{m_{1} \times m_{1}}, R_{22} \in \mathbb{R}^{m_{2} \times m_{2}}$ are positive definite. The noncooperative aspect of the game implies that the players are assumed to not collaborate to attain their goal. The model and the objective functions are assumed to be common knowledge. An admissible set of control actions $\left(\boldsymbol{u}_{1}^{*}, \boldsymbol{u}_{2}^{*}\right)$ is a Nash equilibrium for a two-player game in Eqs. (15)(16) if, for all admissible $\left(\boldsymbol{u}_{1}, \boldsymbol{u}_{2}\right)$, the following inequalities hold:

$$
\begin{aligned}
& J_{1}\left(\boldsymbol{x}_{0}, \boldsymbol{u}_{1}^{*}, \boldsymbol{u}_{2}^{*}\right) \leq J_{1}\left(\boldsymbol{x}_{0}, \boldsymbol{u}_{1}, \boldsymbol{u}_{2}^{*}\right) \\
& J_{2}\left(\boldsymbol{x}_{0}, \boldsymbol{u}_{1}^{*}, \boldsymbol{u}_{2}^{*}\right) \leq J_{1}\left(\boldsymbol{x}_{0}, \boldsymbol{u}_{1}^{*}, \boldsymbol{u}_{2}\right)
\end{aligned}
$$

Nash equilibrium is defined such that there is no incentive for any unilateral deviation by anyone of the players. In general, the Nash equilibrium is not unique. It is easily verified that, whenever $\left(\boldsymbol{u}_{1}^{*}, \boldsymbol{u}_{2}^{*}\right)$ is a Nash equilibrium for a game with cost functions $J_{1}, J_{2}$ respectively, it is also a Nash equilibrium for the game with cost functions $\alpha_{1} J_{1}, \alpha_{2} J_{2} \forall \alpha_{1}, \alpha_{2}>0$. 


\section{Two-Player Game for Rendezvous in Orbit}

In this section the rendezvous problem between two active deputy spacecraft in a LVLH frame is formulated as a cooperative and noncooperative nonzero-sum differential game. The relative equations of motion of Deputy 1 and Deputy 2 are written with respect to the same chief by using the NERM. The chief becomes, in this context, a virtual chief in order to have a reference orbit during the maneuver.

The SDRE method [9] is used to extend the application of the LQ game theory to the rendezvous problem between two active spacecraft in the LVLH frame.

\section{A. State Dependent Coefficient Parametrization}

From section II, the nonlinear dynamics of Deputy 1 and Deputy 2 can be written as:

$$
\begin{aligned}
& \dot{\boldsymbol{x}}_{1}=f\left(\boldsymbol{x}_{1}\right)+B_{1} \boldsymbol{u}_{1}(t) \\
& \dot{\boldsymbol{x}}_{2}=f\left(\boldsymbol{x}_{2}\right)+B_{2} \boldsymbol{u}_{2}(t)
\end{aligned}
$$

defining the game state vector as $\boldsymbol{x}=\boldsymbol{x}_{1}-\boldsymbol{x}_{2}$ we can write:

$$
\dot{\boldsymbol{x}}=f\left(\boldsymbol{x}_{1}\right)-f\left(\boldsymbol{x}_{2}\right)+B_{1} \boldsymbol{u}_{1}(t)-B_{2} \boldsymbol{u}_{2}(t)
$$

by introducing the following time-varying parameters:

$$
\begin{aligned}
& \left\{\begin{array}{l}
\alpha_{x}=\mu\left(r+x_{2}\right)\left(\frac{\left(r_{d_{1}}^{2}+r_{d_{1}} r_{d_{2}}+r_{d_{2}}^{2}\right)}{r_{d_{2}}^{3} r_{d_{1}}^{3}\left(r_{d_{1}}+r_{d_{2}}\right)}\right) \\
\alpha_{y}=\mu y_{2} \frac{\left(r_{d_{1}}^{2}+r_{d_{1}} r_{d_{2}}+r_{d_{2}}^{2}\right)}{r_{d_{2}}^{3} r_{d_{1}}^{3}\left(r_{d_{1}}+r_{d_{2}}\right)} \\
\alpha_{z}=\mu z_{2} \frac{\left(r_{d_{1}}^{2}+r_{d_{1}} r_{d_{2}}+r_{d_{2}}^{2}\right)}{r_{d_{2}}^{3} r_{d_{1}}^{3}\left(r_{d_{1}}+r_{d_{2}}\right)}
\end{array}\right. \\
& \left\{\begin{array}{l}
\beta_{x}=\left(2 r+x_{1}+x_{2}\right) \\
\beta_{y}=\left(y_{1}+y_{2}\right) \\
\beta_{z}=\left(z_{1}+z_{2}\right)
\end{array}\right.
\end{aligned}
$$

we can construct a state dependent coefficient (SDC) parametrization as:

$$
\boldsymbol{x}=A(\boldsymbol{x}) \boldsymbol{x}+B_{1}(\boldsymbol{x}) \boldsymbol{u}_{1}(t)+B_{2}(\boldsymbol{x}) \boldsymbol{u}_{2}(t)
$$

The state and input matrices are given by:

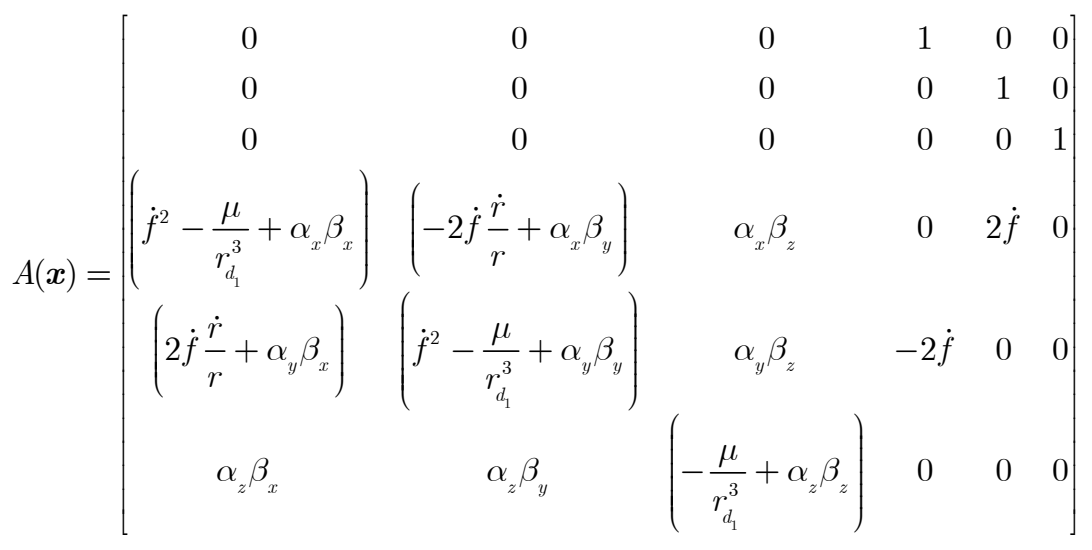

American Institute of Aeronautics and Astronautics 


$$
B_{1}(\boldsymbol{x})=\left[\begin{array}{c}
0_{3 \times 3} \\
\frac{1}{m_{1}(t)} I_{3 \times 3}
\end{array}\right], \quad B_{2}(\boldsymbol{x})=\left[\begin{array}{c}
0_{3 \times 3} \\
-\frac{1}{m_{2}(t)} I_{3 \times 3}
\end{array}\right]
$$

The mass variation of the deputies has been introduced according to the standard mathematical model: $\dot{m}_{i}=u_{i} /\left(g_{0} I_{s p}\right)$, where $I_{s p}$ is the specific impulse and $g_{0}$ is the gravity acceleration at the sea level. Fig. 1 shows a general configuration for our problem.

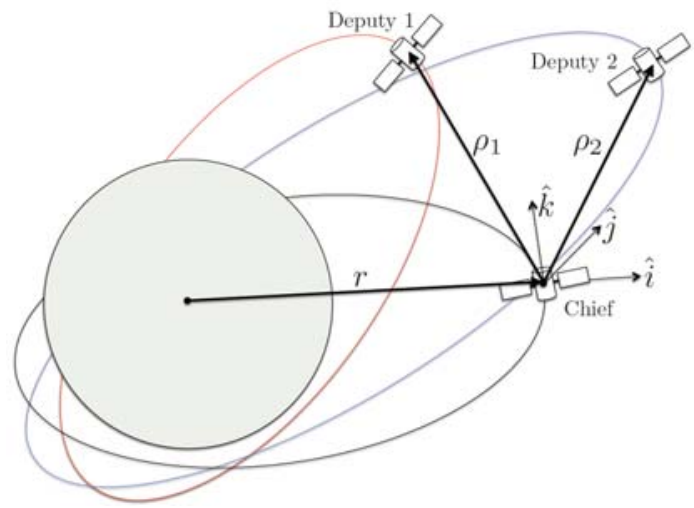

Fig. 1: Two-Player Dynamics in the LVLH Frame

\section{B. Nonlinear Cooperative Control Strategy}

The state feedback cooperative control law obtained from Eq. (13) is given by:

$$
\left\{\begin{array}{l}
\boldsymbol{u}_{c}(\boldsymbol{x}, \alpha)=-R_{c}(\boldsymbol{x}, \alpha)^{-1} B_{c}^{\mathrm{T}}(\boldsymbol{x}) P(\boldsymbol{x}, \alpha) \boldsymbol{x}(t) \\
B_{c}(\boldsymbol{x})=\left[\begin{array}{ll}
B_{1}(\boldsymbol{x}) & B_{2}(\boldsymbol{x})
\end{array}\right]
\end{array}\right.
$$

$P(\boldsymbol{x}, \alpha)$ is the unique, symmetric, positive-definite pointwise stabilizing solution of the following continous time state-dependent algebraic Riccati equation (ARE):

$$
P(\boldsymbol{x}, \alpha) A(\boldsymbol{x})+A^{\mathrm{T}}(\boldsymbol{x}) P(\boldsymbol{x}, \alpha)-P(\boldsymbol{x}, \alpha) B_{c}(\boldsymbol{x}) R_{c}(\boldsymbol{x}, \alpha)^{-1} B_{c}^{\mathrm{T}}(\boldsymbol{x}) P(\boldsymbol{x}, \alpha)+Q(\boldsymbol{x}, \alpha)=0
$$

The resulting cooperative closed-loop controlled trajectory for the two players differential game is

$$
\dot{\boldsymbol{x}}(t)=\left[A(\boldsymbol{x})-B_{c}(\boldsymbol{x}) R_{c}^{-1}(\boldsymbol{x}, \alpha) B_{c}^{\mathrm{T}}(\boldsymbol{x}) P(\boldsymbol{x}, \alpha)\right] \boldsymbol{x}(t)
$$

where the state-dependent closed-loop matrix is pointwise Hurwitz $\forall \boldsymbol{x}$. In the next simulations, a combination of Schur and Kleimann recursive algorithms [10] is used to solve the Eq. (23) at each time step.

\section{Nonlinear Noncooperative Control Strategy}

The nonzero-sum Nash control law for Deputy 1 and Deputy 2 are respectively:

$$
\begin{aligned}
& \boldsymbol{u}_{1}(t)=-R_{11}^{-1}(\boldsymbol{x}) B_{1}^{\mathrm{T}} P_{1}(\boldsymbol{x}) \boldsymbol{x}(t) \\
& \boldsymbol{u}_{2}(t)=-R_{22}^{-1}(\boldsymbol{x}) B_{2}^{\mathrm{T}} P_{2}(\boldsymbol{x}) \boldsymbol{x}(t)
\end{aligned}
$$


where $\left(P_{1}(\boldsymbol{x}), P_{2}(\boldsymbol{x})\right)$ are the pointwise stabilizing solutions of the state-dependent coupled AREs:

$$
\begin{aligned}
& \left(A(\boldsymbol{x})-S_{2}(\boldsymbol{x}) P_{2}(\boldsymbol{x})\right)^{\mathrm{T}} P_{1}(\boldsymbol{x})+P_{1}(\boldsymbol{x})\left(A(\boldsymbol{x})-S_{2}(\boldsymbol{x}) P_{2}(\boldsymbol{x})\right)-P_{1}(\boldsymbol{x}) S_{1}(\boldsymbol{x}) P_{1}(\boldsymbol{x})+Q_{1}(\boldsymbol{x})+P_{2}(\boldsymbol{x}) S_{21}(\boldsymbol{x}) P_{2}(\boldsymbol{x})=0 \\
& \left(A(\boldsymbol{x})-S_{1}(\boldsymbol{x}) P_{1}(\boldsymbol{x})\right)^{\mathrm{T}} P_{2}(\boldsymbol{x})+P_{2}(\boldsymbol{x})\left(A(\boldsymbol{x})-S_{1}(\boldsymbol{x}) P_{1}(\boldsymbol{x})\right)-P_{2}(\boldsymbol{x}) S_{2}(\boldsymbol{x}) P_{2}(\boldsymbol{x})+Q_{2}(\boldsymbol{x})+P_{1}(\boldsymbol{x}) S_{12}(\boldsymbol{x}) P_{1}(\boldsymbol{x})=0
\end{aligned}
$$

the following shorthand notation is used in Eqs. (26):

$$
\left\{\begin{array}{l}
S_{1}(\boldsymbol{x})=B_{1}(\boldsymbol{x}) R_{11}^{-1}(\boldsymbol{x}) B_{1}^{\mathrm{T}}(\boldsymbol{x}) \\
S_{2}(\boldsymbol{x})=B_{2}(\boldsymbol{x}) R_{22}^{-1}(\boldsymbol{x}) B_{2}^{\mathrm{T}}(\boldsymbol{x}) \\
S_{12}(\boldsymbol{x})=B_{1}(\boldsymbol{x}) R_{11}^{-1}(\boldsymbol{x}) R_{21} R_{11}^{-1} B_{1}^{\mathrm{T}}(\boldsymbol{x}) \\
S_{21}(\boldsymbol{x})=B_{2}(\boldsymbol{x}) R_{22}^{-1}(\boldsymbol{x}) R_{12} R_{22}^{-1} B_{2}^{\mathrm{T}}(\boldsymbol{x})
\end{array}\right.
$$

The state-dependent closed-loop trajectory for the Deputies choosing a Nash strategy is then:

$$
\dot{\boldsymbol{x}}(t)=\left[A(\boldsymbol{x})-B_{1}(\boldsymbol{x}) R_{11}^{-1}(\boldsymbol{x}) B_{1}^{\mathrm{T}}(\boldsymbol{x}) P_{1}(\boldsymbol{x})-B_{2}(\boldsymbol{x}) R_{22}^{-1}(\boldsymbol{x}) B_{2}^{\mathrm{T}}(\boldsymbol{x}) P_{2}(\boldsymbol{x})\right] \boldsymbol{x}(t)
$$

The solution of the coupled Riccati equations in Eq. (26) requires numerical iteration [11], [12]. The iterative procedure is based on a sequential stepwise decoupling, yielding the solution of two standard equations at each iteration. Two stopping conditions are used: the first is the required accuracy of the solution, while the second limits the maximum number of iterations in order to have an acceptable execution time.

\section{Simulation Results}

In this section we present a sample numerical simulation in order to compare both cooperative and noncooperative approaches. A rendezvous cooperative maneuver scenario was considered, where both spacecraft are controlled and guided in order to match the same position and velocity at final time. In this scenario, the performance of the cooperative and noncooperative differential game strategies were evaluated and compared.

The control law performance were evaluated over one chief's orbit period $\mathrm{T}$, and numerical integration used a 4th order Runge- Kutta integration algorithm, with step size of $T$ / 1000 . For performance comparison consistency, the noncooperative control law was evaluated taking into account the case of the Identical Goal Game [13] that occurs when two players cooperate in order to minimize the same performance functions given in Eq. (15).

The final distance between the deputies $\rho(T)=\left\|\boldsymbol{\rho}_{1}(T)-\boldsymbol{\rho}_{2}(T)\right\|$, the total control usage $\Delta v_{i}$ with $i=1,2$, and the maneuver time to reach the proximity operations range, $10<\rho<100 \mathrm{~m}$, were the performance metrics used for comparison.

The initial conditions in the LVLH frame are (units in $\mathrm{Km}$ and $\mathrm{Km} / \mathrm{sec}$ ):

$$
\begin{aligned}
& x_{1}(0)=\left[\begin{array}{llllllll}
-2200 & 0 & 0 & 0 & 4.359 & 2.071
\end{array}\right]^{\mathrm{T}} \\
& x_{2}(0)=\left[\begin{array}{llllll}
-10.5146 & 17.5431 & -12.2825 & -4.3374 \cdot 10^{-5} & 0.0198 & 8.4732 \cdot 10^{4}
\end{array}\right]^{\mathrm{T}}
\end{aligned}
$$

For Deputy 1 and Deputy 2 respectively. The chief's orbital parameters correspond to an elliptical orbit with the following orbital parameters:

$$
\left\{\begin{array}{l}
e=0.3 \\
a=11,000 \mathrm{Km} \\
i=70^{0} \\
\Omega=45^{0} \\
\omega=0 \\
f\left(t_{0}\right)=0
\end{array}\right.
$$

8

American Institute of Aeronautics and Astronautics 
The performance of the two control strategies are shown in Table A.

Table A. Control Effort (m/sec), Final Distance (M), Maneuver Time (in Periods)

\begin{tabular}{cccccccc}
\hline \multicolumn{3}{c}{ Cooperative Game } & \multicolumn{4}{c}{ Noncoopeative Game } \\
\hline$\Delta v_{1}$ & $\Delta v_{2}$ & $\rho(T)$ & $t_{m}$ & $\Delta v_{1}$ & $\Delta v_{2}$ & $\rho(T)$ & $t_{m}$ \\
\hline 4134 & 2685 & 16.86 & 0.786 & 3521 & 2446 & 24.86 & 0.836 \\
\hline
\end{tabular}

The trajectories in the LVLH frame are shown in Fig. 2.
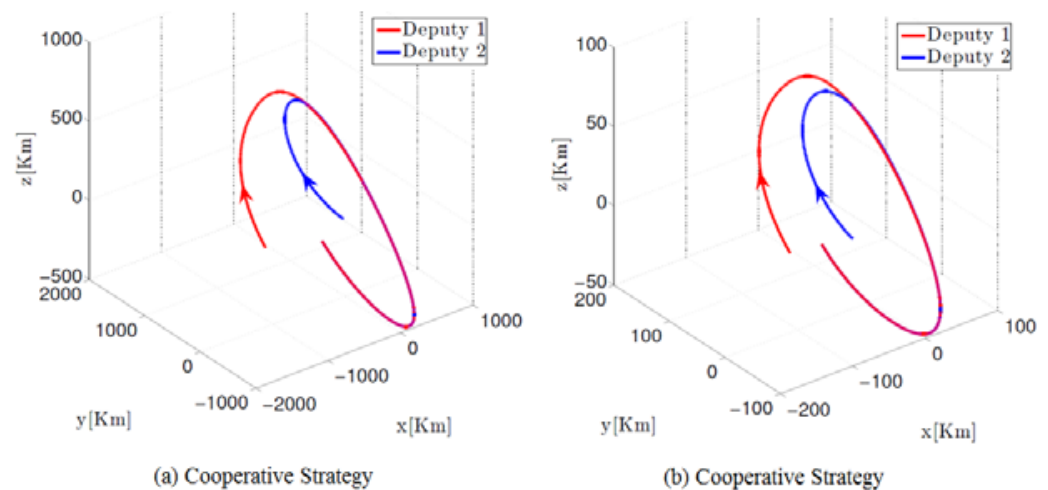

(b) Cooperative Strategy

Figure 2. Spacecraft Trajectories using initial Conditions from Eq. (29)

The cooperative control law requires greater control effort, and the final distance between Deputy 1 and Deputy 2 is smaller in the cooperative case. The two deputies reach the same orbit both in cooperative and in the noncooperative cases. From the results, cooperation appears better, although a noncooperative approach yields good results, and could be used in the case of communications failures between the spacecraft.

\section{Multiplayer Motion Synchronization}

The study of differential games involving multiple players is somewhat limited due to the difficulty in the mathematical formulation and formal treatment. In the literature, most of the studies on the multiplayer differential games are concentrated on the multiple pursuers and evaders scenario, see for instance [14], [15], [16].

A multiplayer differential game is considered in this section as a possible approach to the synchronization of the relative motion among multiple spacecraft. A general nonzero-sum differential game with $n$ players is decomposed into $n-1$ sequential games in time, between two players that are closely engaged. In this case the overall control structure consists of a higher level strategy responsible for establishing an a priori engagement scheme among the players, and a lower level strategy given by a two-player differential game. The latter can be viewed as a natural hierarchical structure in decision-making between the players.

Fig. 3 shows an example of an open loop sequential multiplayer strategy. To achieve sequentiality, once a twoplayer game ends, their common state (for example their common center of mass) $\boldsymbol{x}_{c m}$ becomes a single new player for the next game. The procedure outlined above is of course heuristical, and suboptimal with respect to a simultaneous multiplayer game. 


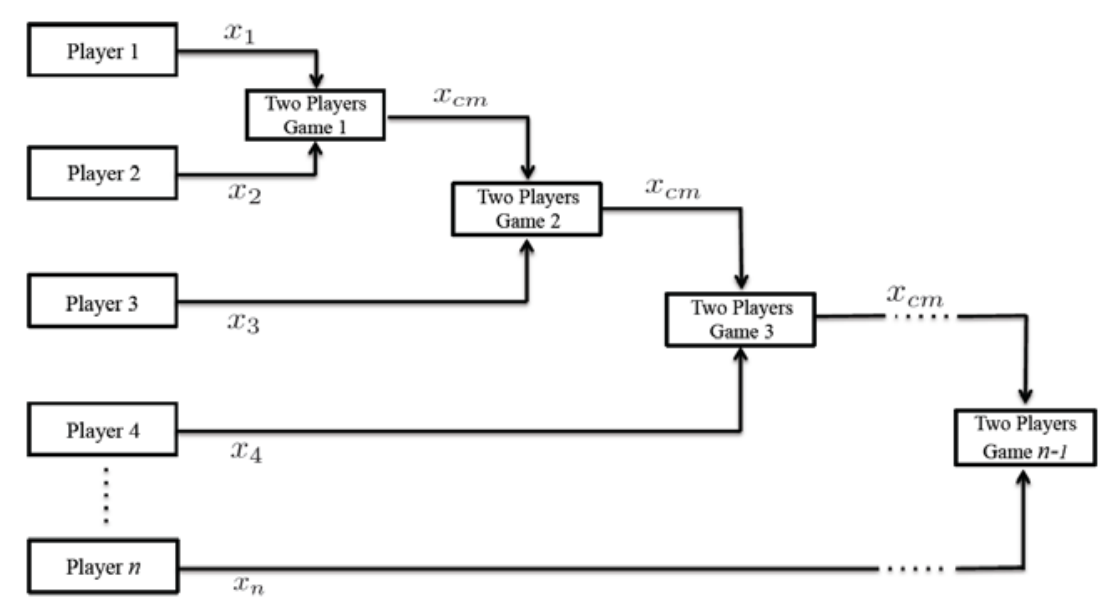

Figure 3. High Level sequential Multiplayer Control Management Strategy

\section{A. On-Orbit Autonomous Assembly Mission}

From the space application point-of-view, the multiplayer differential game can be viewed as a strategy for onorbit assembly of modular spacecraft. The next-generation of human space exploration programs, in fact, should be designed for both sustainability and affordability. The cost of such programs can be reduced through the use of flexible infrastructures supporting various aspects of manned spaceflight.

On-orbit assembly of separate launched spacecraft modules, is an important component of the infrastructure enabling human access to space. New technologies and architecture concepts are being developed that make the robotic autonomous assembly more feasible today with respect to the past. Specifically, the modular spacecraft design is a key enabling concept for robotic on-orbit and rendezvous assembly, because it reduces the complexity of the task. Assembly of separate modules, by docking them together, is simpler than attaching individual trusses and solar panels, or assemblage large mirrors in space.

In a classical strategy for on-orbit assembly and rendezvous, the chief or target spacecraft is a passive vehicle that does not apply any control force while following its trajectory and the second spacecraft is an active vehicle, which is controlled in such a way to meet the target spacecraft. In a cooperative maneuver, both spacecraft are controlled and guided to match the same position and velocity. Two possible strategies using multiple spacecraft are illustrated in Fig. 4.

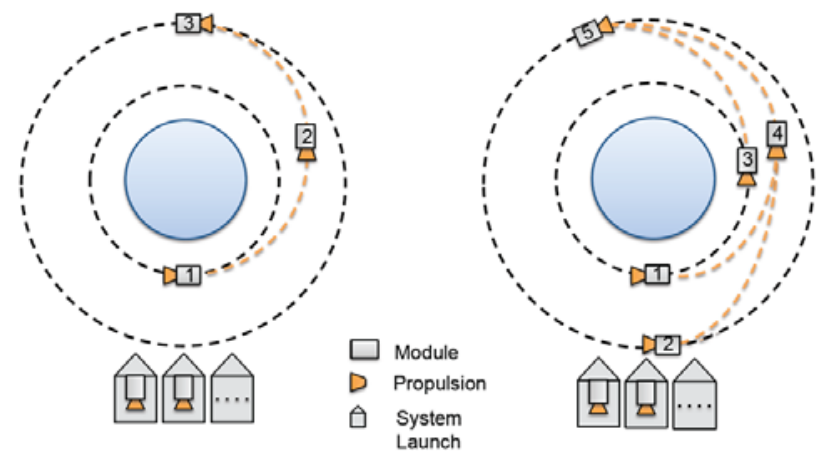

Figure 4. Self Assembly Strategies

A numerical simulation campaign was performed using the cooperative non-zero sum differential game approach described earlier. Simulations were performed for the nonlinear and linear cases. A comparison between the trajectories obtained using different linear approximations, such as HCW, LERM and VC equations, is shown.

In the simulations, the sample time was set to $T / 1000$, and $4^{\text {th }}$ order Runge-Kutta integration algorithm used. The number of spacecraft considered for the sequential game strategy test is 4 . Each spacecraft, at the beginning of 
the engagement, has a fuel mass of $m_{\text {prop }}=90 \mathrm{Kg}$ and a specific impulse of $I_{s p}=310$ seconds. The mass variation as a function of the propellant consumption was also considered in the simulations.

The docking operation range used to determine the final state of each two-player game was $\rho<10 \mathrm{~m}$ [17] in thesis], where $\rho$ is the distance between the two deputies. At the end of each game, the center of mass of the two deputies is considered as the common state, and given by:

$$
\boldsymbol{x}_{c m}=\frac{m_{1}\left(t_{f}\right) \boldsymbol{x}_{1}+m_{2}\left(t_{f}\right) \boldsymbol{x}_{2}}{m_{1}\left(t_{f}\right)+m_{2}\left(t_{f}\right)}
$$

where $m_{i}\left(t_{f}\right)$, with $i=1,2$ is the final mass of the deputies, and the time $t_{f}$ is the final time of the two-player game. The engagement was optimized by selecting the sequence of the two-player game in order to minimize the maneuver time in each game. The set of weighting matrices were selected for the 3 sequential games to minimize the propellant consumption. The Pareto parameter $\alpha$ was chosen for each game to yield the same mass between the deputies at the final time $t_{f}$. The mission length was simulated over 2 chief's orbital periods and only in-plane motion is shown in Fig. 5. The following Cartesian initial conditions were used for the four spacecraft (units are in $\mathrm{m}$ and $\mathrm{m} / \mathrm{sec})$ :

$$
\begin{aligned}
& \boldsymbol{x}_{1}(0)=\left[\begin{array}{llll}
-75.0003 & 67.4992 & 0 & 0.1710
\end{array}\right]^{T} \\
& \boldsymbol{x}_{2}(0)=\left[\begin{array}{llll}
-150.0013 & 134.9970 & 0 & 0.3419
\end{array}\right]^{T} \\
& \boldsymbol{x}_{3}(0)=\left[\begin{array}{llll}
-225.0030 & 202.4932 & 0 & 0.5129
\end{array}\right]^{T} \\
& \boldsymbol{x}_{4}(0)=\left[\begin{array}{llll}
-300.0054 & 269.988 & 0 & 0.6839
\end{array}\right]^{T}
\end{aligned}
$$

The accuracy of the simulations results were then evaluated by computing the norm of the difference between $\boldsymbol{\rho}(t)$ of the VC and HCW with respect to $\boldsymbol{\rho}_{L E R M}(t)$

$$
\varepsilon(t)=\left\|\boldsymbol{\rho}_{i}(t)-\boldsymbol{\rho}_{L E R M}(t)\right\|, i=V C, H C W
$$

where $\boldsymbol{\rho}(t)$ is the distance between the two deputies during each game. The four deputies trajectories XY projections are shown in Fig. 5.

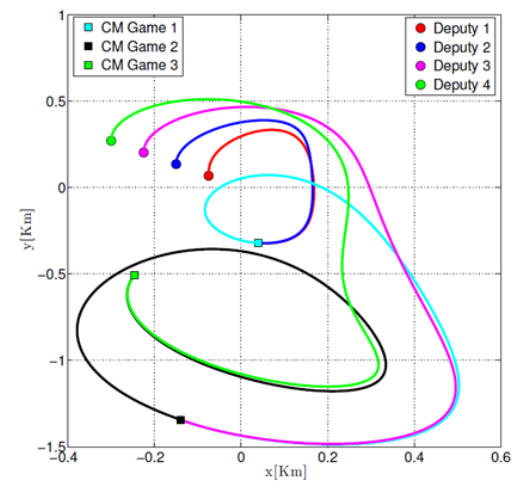

VC Cooperative sequential Game

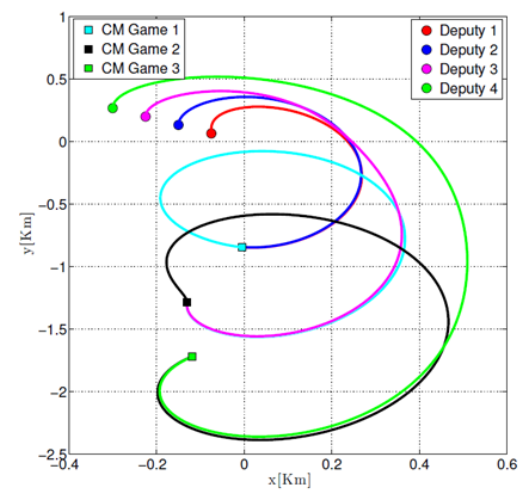

HCW Cooperative sequential Game

Figure 5. Linear Cooperative sequential Games Trajectories $(e=0.1)$

For comparison (note the scale differences) the same simulations using LERM are shown in Fig. 6. The same figure presents the cumulative errors $\varepsilon$ in meters during the 3-game sequence. The VC approach yields better performance, since the HCW uses nominal circular orbits. 


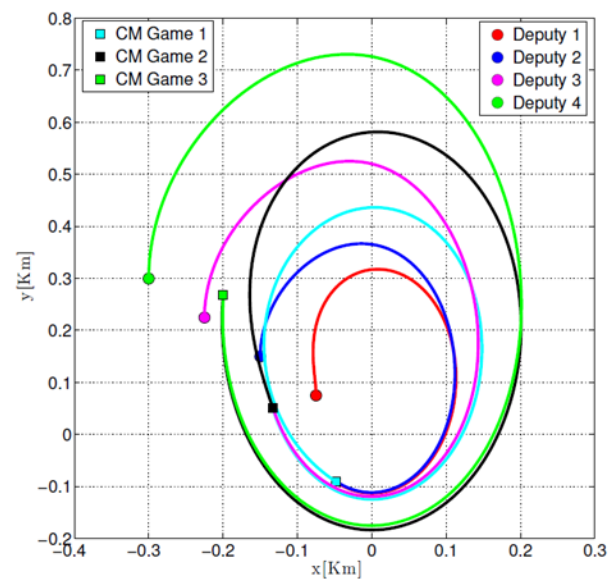

\begin{tabular}{lcccccc}
\multicolumn{4}{c}{ Average Error (meters) } \\
\multicolumn{4}{c}{ Game 1 } & \multicolumn{2}{c}{ Game 2 } & \multicolumn{2}{c}{ Game 3 } \\
VC & HCW & VC & HCW & VC & HCW \\
0.49 & 0.91 & 34.38 & 83.38 & 149.73 & 338.94
\end{tabular}

LERM Cooperative sequential Game

Figure 6. Linear Sequential Games using LERM

\section{Conclusion}

A nonzero-sum differential game structure was applied to cooperative maneuvers for two active spacecraft during rendezvous. A comparison between a cooperative Pareto game and noncooperative Nash solution was carried out. Using a two- player LQ differential game approach, nonlinear control laws were developed using a state-dependent coefficient parametrization of the game dynamics and the SDRE method. The cooperative and noncooperative control laws have shown to yield similar performance, in terms of control effort and final distance between the deputies. The disadvantage of the noncooperative game control law is a higher computational time, and numerical convergence due to the solution of coupled Riccati equations. The cooperative game controller was also tested for relative motion synchronization among many deputies using a suboptimal sequential game approach. A comparison among the trajectories obtained by using the HCW, VC and LERM equations, indicated the feasibility of the approach, and the better performance of a virtual chief model, especially if we consider non circular orbits.

\section{References}

[1] H., P., Schaub, J., L., Junkins. Analytical mechanics of space systems. AIAA Education Series, Second Edition. AIAA Reston, VA, 2009.

[2] R., E., Sherrill, A. J. Sinclair, T. A. Lovell, "Virtual Chief Generalization of Hill-Clohessy-Wiltshire to Elliptic Orbits", AIAA Journal of Guidance, Control, and Dynamics (2014), Vol. 1, pp. 1-5.

[3] R., Isaacs. Differential Games, John Wiley and Sons, 1965.

[4] J., Eengwerda. Q dynamic optimization and differential games. John Wiley \& Sons, 2005.

[5] M., Pavone, B., Acikmese, I., Nesnas and J. Starek, Spacecraft Autonomy Challenges for Next Generation Space Missions. Springer Lecture Notes in Control and Information Sciences, 2014.

[6] D., P., Scharf, F., Y., Hadaegh, S., R., Ploen,” A survey of spacecraft formation flying guidance and control. part ii: control”, Proceedings of the 2004 American Control Conference, ACC04, Vol.4, pp. 2976-2985.

[7] J., Nash, “The bargaining problem”, Econometrica, Journal of Econometric Society, (1950), pp. 155-162.

[8] J., Nash, “Two-Person cooperative games”, Econometrica, Journal of Econometric Society, (1953), pp. 128-140.

[9] T., Cimen, "A survey of state-dependent Riccati equations in nonlinear optimal feedback control synthesis", AIAA Journal of Guidance, Control, and Dynamics, (2012), Vol. 35, No. 4, pp. 1025-1047.

[10] D., Kleinman, “On an iterative techniquefor Riccati equations computations”, IEEE Transactions on Automatic Control (1968), Vol. 13, No. 1, pp. 114-115.

[11] J. Engwerda, Algorithms for computing Nash equilibria in deterministic LQ games”, Computational Management Science (2007), Vol. 4, No. 2, pp. 113-140.

[12] L. Cherfi, Y. Chitour, H., Habou-Chandil, “A new algorithm for solving coupled algebraic Riccati equations”, IEEE Conference on Computational Intelligence for Modeling, Control, and Automation (2005), Vol. 1, pp. 83-88.

[13] A., W., Starr, Y., C., Ho, "Non-zero sum differential games”, Journal of Optimization Theory and Applications (1969), Vol. 3, No. 3, pp. 184-206.

[14] Z., S., Cai, L., N., Sun, H., B., Gao, “A novel hierarchical decomposition for multi-player pursuit evavion differential game with superior evader”, Proceedings of the 1st Summit on Genetic and Evolutionary Computation (2009), pp. 795-798.

[15] R., Jensen, "Efficient method for computing strategies for successive pursuit differential games", Northeastern University, MAsterès Thesis (2014). 
[16] D., Li, J., B., Cruz, C. Schumacher, “A hierarchical approach to multi-player pursuit-evasion differential game”, IEEE Control and Decision Conference (2005), pp. 5674-5679.

[17] J., R., Wertz, R., Bell, “Autonomous rendezvous and docking technologies: status and prospects”, Aerosense 2003, pp. 2030. 